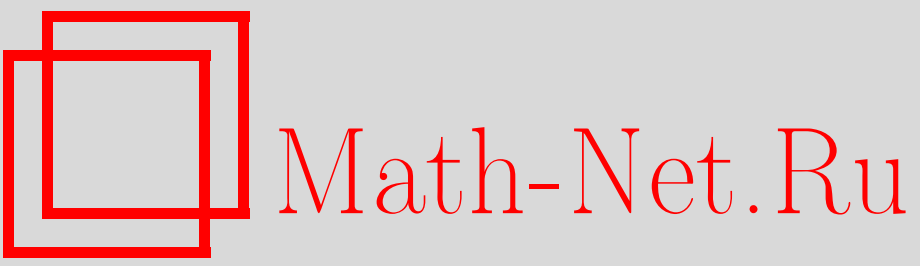

Г. Л. Литвинов, В. П. Маслов, Идемпотентная математика: принцип соответствия и его компьютерные приложения, УМH, 1996, том 51, выпуск 6, 209-210

DOI: https://doi.org/10.4213/rm1031

Использование Общероссийского математического портала Math-Net.Ru подразумевает, что вы прочитали и согласны с пользовательским соглашением

http://www . mathnet.ru/rus/agreement

Параметры загрузки:

IP : 3.80 .181 .102

26 апреля 2023 г., 12:34:53 


\title{
ИДЕМПОТЕНТНАЯ МАТЕМАТИКА: ПРИНЦИП СООТВЕТСТВИЯ И ЕГО КОМПЬЮТЕРНЫЕ ПРИЛОЖЕНИЯ
}

\author{
Г. Л. Литвинов, В. П. МАслов
}

1. Введение. Имеется (эвристическое) соответствие между важными, полезными и интересными конструкциями и результатами традиционной математики над полями и аналогичными конструкциями и результатами над полукольцами с идемпотентным сложением в духе принципа соответствия Н. Бора в квантовой механике. Последовательное применение идемпотентного принципа соответствия приводит к разнообразным результатам, в том числе к методике конструирования унифицированных алгоритмов для научно-технических расчетов и программной и аппаратной реализации этих алгоритмов (например, методике патентования компьютерных устройств).

2. Идемпотентный принцип соответствия. Пусть $\mathbb{R}$ - поле действительных чисел, $\mathbb{R}_{+}-$полукольцо неотрицательных чисел (относительно сложения и умножения). Замена переменных $x \mapsto u=h \ln x$ определяет отображение $\Phi_{h}: \mathbb{R}_{+} \rightarrow A=\mathbb{R} \cup\{-\infty\}$. Перенесем сложение и умножение из $\mathbb{R}$ в $A$ с помощью $\Phi_{h}$, т.е. положим $u \oplus_{h} v=h \ln (\exp (u / h)+\exp (v / h))$, $u \odot v=u+v, \mathbf{0}=-\infty=\Phi_{h}(0), \mathbf{1}=0=\Phi_{h}(1)$. Легко проверить, что $u \oplus_{h} v \rightarrow \max \{u, v\}$ при $h \rightarrow 0$, а множество $A$ является полукольцом относительно операций сложения $u \oplus v=\max \{u, v\}$ и умножения $u \odot v=u+v$ с нулем $\mathbf{0}=-\infty$ и единицей $\mathbf{1}=0$. Обозначим это полукольцо через $\mathbb{R}_{\max }$; оно идемпотентно, т.е. $u \oplus u=u$ для всех его элементов. Имеется очевидная аналогия с квантованием, и параметр $h$ играет роль постоянной Планка, т.е. $\mathbb{R}_{+}$(или $\mathbb{R}$ ) можно рассматривать как "квантовый объект", $a \mathbb{R}_{\max }$ - как результат "деквантования" этого объекта. Аналогичная процедура приводит к полукольцу $\mathbb{R}_{\min }=\mathbb{R} \cup\{+\infty\}$ с операциями $\oplus=\min$, $\odot=+$; в этом случае $\mathbf{0}=+\infty, \mathbf{1}=0$. Полукольца $\mathbb{R}_{\max }$ и $\mathbb{R}_{\min }$ изоморфны. Идемпотентное полукольцо $\mathbb{R} \cup\{-\infty\} \cup\{+\infty\}$ с операциями $\oplus=\max , \odot=\min$ можно получить как результат "вторичного деквантования" по отношению к $\mathbb{R}$ (или $\mathbb{R}_{+}$). Можно указать десятки интересных примеров неизоморфных идемпотентных полуколец и стандартные конструкции, позволяющие строить из них новые идемпотентные полукольца, см. [1]-[3].

Пусть $A$ - произвольное полукольцо с идемпотентным сложением $\oplus$ (сложение всегда коммутативно), умножением $\odot$, нулем $\mathbf{0}$ и единицей $\mathbf{1}$, снабженное стандартнылм частичныц порядком: $a<b$, если (и только если) $a \oplus b=b$. Аналогом числовой функции является отображение $X \rightarrow A$, где $X$ - любое множество. Функции со значениями в $A$ можно поточечно складывать, умножать и умножать на элементы из $A$. Аналогом линейного функционального пространства является множество $A$-значных функций, выдерживающее сложение функций и умножение на элементы из $A$, т.е. $A$-полумодуль. Примером является $A$-полумодуль $B(X, A)$ ограниченных (в смысле стандартного порядка на $A$ ) функций $X \rightarrow A$. Для функции $\varphi \in B(X, A)$ идемпотентный аналог интеграла определяется формулой

$$
I(\varphi)=\int_{X}^{\oplus} \varphi(x) d x=\sup _{x \in X} \varphi(x) .
$$

$A$-мера на $X$ определяется равенством $m_{\psi}(B)=\sup _{x \in B} \psi(x)$, где $B \subset X, \psi \in B(X, A)$. Интеграл по этой мере определяется формулой

$$
I_{\psi}(\varphi)=\int_{X}^{\oplus} \varphi(x) d m_{\psi}=\int_{X}^{\oplus} \varphi(x) \odot \psi(x) d x=\sup _{x \in X} \varphi(x) \odot \psi(x) .
$$

Функционалы $I(\varphi)$ и $I_{\psi}(\varphi)$ линейны над $A$, их значения соответствуют предельным значениям аналогов сумм Лебега (или Римана). Формула для $I_{\psi}(\varphi)$ определяет идемпотентное скалярное произведение функций $\psi$ и $\varphi$. Используя идемпотентное интегрирование, можно строить различные функциональные пространства и идемпотентную версию теории обобщенных функций [1]-[3].

Работа выполнена при финансовой поддержке Российского фонда фундаментальных исследований (грант № 96-01-01544). 
Аналогия между идемпотентными и вероятностными мерами приводит к примечательным параллелям между теорией вероятностей и теорией оптимизации. При этом уравнение Чепмена-Колмогорова соответствует уравнению Беллмана (P. Del Moral, см. его Приложение к [3]). Можно указать и много других идемпотентных аналогий (в том числе для основных конструкций и результатов функционального анализа). Например, преобразование Лежандра есть не что иное,

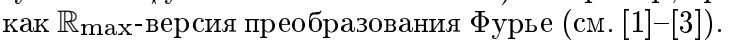

Основные уравнения квантовой теории линейны (принцип суперпозиции). Уравнение Гамильтона-Якоби, т.е. основное уравнение классической механики, нелинейно в обычном смысле. Однако его можно рассматривать как линейное над полукольцом $\mathbb{R}_{\min }$, а различные версии уравнения Беллмана, т.е. основного уравнения теории оптимизации, также являются линейными над идемпотентными полукольцами (см. [1]-[3]). Например, конечномерное стационарное уравнение Беллмана можно представить в форме $X=H \odot X \oplus F$, где $X, H, F$ - матрицы с элементами из идемпотентного полукольца $A$, а решение $X$ определяется из этого уравнения при заданных $H$ и $F[4]$. В частности, стандартные задачи динамического программирования и хорошо известная задача о кратчайшем пути соответствуют случаю $A=\mathbb{R}_{\max }$. В [4] указано, что основные оптимизационные алгоритмы на конечных графах соответствуют стандартным методам решения системы линейных уравнений указанного типа (над полукольцами). Алгоритм Беллмана для задачи о кратчайшем пути соответствует версии метода Якоби, а алгоритм Форда соответствует итерационному методу Гаусса-Зейделя и т. п.

3. Принцип соответствия для алгоритмов и их компьютерных реализаций [5]. Идемпотентные аналоги стандартных численных алгоритмов часто являются важными и могут быть использованы систематически, например при решении оптимизационных задач. Особенно важны алгоритмы линейной алгебры, поскольку стандартные бесконечномерные линейные задачи над полукольцами сводятся к их конечномерным (или конечным) аппроксимациям, а нелинейные алгоритмы обычно приближаются линейными. Известные методы распараллеливания алгоритмов линейной алгебры работают и для их идемпотентных аналогов. Стандартные численные алгоритмы реализованы в виде многочисленных технических устройств (процессоров). Такое устройство может являться прототипом для идемпотентного аналога. Для конструирования и патентования такого аналога следует указать прототип и отличия нового устройства от прототипа, что составляет формулу изобретения. Отличия состоят в том, что обычные ариффетические операции над числами заменяются на операции над элементами числового полукольца (с учетом обработки элементов типа $\pm \infty$ ). При этом удачные технические решения переносятся с прототипа на аналог. Простейшим примером является алгоритм вычисления скалярного произведения двух векторов. Его полукольцевая версия очевидна и основана на формуле $(x, y)=\left(x_{1} \odot y_{1}\right) \oplus\left(x_{2} \odot y_{2}\right) \oplus \cdots \oplus\left(x_{n} \odot y_{n}\right)$. Такое вычисление стандартно для алгоритмов оптимизации (и полуколец типа $\mathbb{R}_{\max }$ ).

Программная реализация "полукольцевых" алгоритмов может быть особенно гибкой. Программные модули могут оперировать с абстрактными (и переменными) операциями и типами данных, что особенно удобно для работы с нечисловыми полукольцами. При этом конкретные значения операций определяются типом входных данных. Для такой реализации удобна современная техника объектно ориентированного программирования, включая специальные языки программирования, например, $\mathrm{C}^{++}$.

\section{СПИСОК ЛИТЕРАТУРЫ}

[1] Maslov V. P., Samborskiü S. N., Eds. Idempotent analysis. V. 13. Providence: AMS Adv. Sov. Math., 1992. [2] Маслов В.П., Колокольцов В.Н. Идемпотентный анализ и его применение в оптимальном управлении. М.: Наука, 1994. [3] Maslov V.P., Kolokoltsov V. N. Idempotent analysis and applications: Kluwer (to appear). [4] Carre B. A. // J. Inst. Math. Appl. 1971. V. 7. P. 273-294. [5] Litvinov G. L., Maslov V. P. Correspondence principle for idempotent calculus and some computer applications // Bures-sur-Yvette. Preprint IHES, 1995. 